Fecha de recepción: abril 2019

Fecha de aceptación: octubre 2019

Versión final: marzo 2020

\section{Diseño y cultura. Huellas japonesas en la Argentina}

\author{
Marcia Veneziani *
}

\begin{abstract}
Resumen: Dado el creciente interés por la cultura japonesa en la Argentina, en el presente ensayo se intenta analizar el vínculo entre los conceptos de instante, vacío, ambigüedad y vulnerabilidad, para relacionarlos con el diseño, el arte, la estética, la filosofía Zen y la naturaleza. El kimono, la puerta característica denominada shöji, sus marcos de madera y papel washi, la poesía Haiku, el blanco y las sombras, son algunos de los ejemplos que se abordan para explicarlo. Resulta esencial además, comprender que también el lenguaje japonés, está indisolublemente ligado al diseño y a su filosofía. Para un japonés hablar y escuchar es un verdadero arte. Es por ello que el escrito aborda la cuestión a partir, no solo de los textos de Kenya Hara, Junichirō Tanizaki y de Jorge Luis Borges, sino que además incluye -necesariamente- un diálogo entre la autora del presente artículo y la filósofa nipona Mami Goda, residente en la Argentina desde hace casi una década.
\end{abstract}

Palabras clave: cultura japonesa - vacío - instante - ambigüedad - vulnerabilidad - diseño - arte - filosofía Zen - Haiku - kimono.

[Resúmenes en inglés y portugués en la página 28]

${ }^{(*)}$ Doctora en Comunicación Social y Licenciada en Publicidad (USAL). Profesora de la Universidad de Palermo en el Área de Investigación y Producción de la Facultad de Diseño y Comunicación. Es autora de los libros La Imagen de la Moda, y Moda, Economía y Sociedad. Además de escribir numerosos ensayos sobre comunicación, ha coordinado publicaciones académicas internacionales con la Universidad de Palermo y la Università di Bologna, y Parsons The New School for Design, entre otras. Especialista en temas de comportamiento de Moda y Consumo. Miembro del Comité Editorial de la Revista ZoneModa Journal, dedicada a los estudios de moda en Italia, Universitá di Bologna, Italia, desde julio de 2013. Directora de las Jornadas de Moda y Pensamiento: "Hipermoda". Fundación Ortega y Gasset Argentina (2015) y miembro del staff del cuerpo académico del Centro de Estudios Latinoamericanos de La Abadía y del Centro de Diplomacia Karina Vilella. Ha sido Profesora Titular en la Maestría en Comercialización y Comunicación Publicitaria de la Universidad del Salvador. También se desempeñó en importantes agencias de publicidad, organizaciones educativas y ha colaborado con diversos medios de comunicación y prensa en temas que tratan sobre su especialidad. Pertenece a la Facultad de Diseño y Comunicación desde el año 2009. Forma parte del Cuerpo Académico del Doctorado en Diseño, de la Maestría en Gestión del Diseño y del Equipo de Evaluación de Proyectos de 
Graduación. Miembro del Plenario de la Comisión de Posgrado DC. Dirige la Línea de Investigación Nuevos Paradigmas en la Enseñanza de la Moda y el Diseño.

Las relaciones diplomáticas entre la Argentina y Japón se iniciaron en 1898, con la firma del Tratado de Amistad, Comercio y Navegación.

El vínculo entre ambos países se fue afianzando debido a la inmigración japonesa en nuestro país. Luego de la Segunda Guerra Mundial, en la cual fueron suspendidas las relaciones bilaterales por la declaración de guerra por parte de Argentina hacia Japón en marzo de 1945, volvieron a restaurarse en el año 1952 con la firma del Tratado de Paz. Como indica en su página web, la Embajada de Japón en la Argentina, ambos países "han firmado un Acuerdo de Migración (1963), un Tratado de Amistad, Comercio y Navegación (1967), un Convenio sobre Cooperación Técnica (1981) y un Convenio Cultural (1981)" (Embajada de Japón en la Argentina, 2018).

A partir del año 2015 que este vínculo comenzó a crecer de modo más perceptible.

En la Argentina se realizan anualmente más de cien actividades ligadas con Japón, por ejemplo: se celebra la fiesta de Japón; se dictan clases de idioma japonés; comida japonesa; animé y cine japonés. Además se difunde la cultura japonesa tradicional, como por ejemplo, la ceremonia del té, ikebana, música y caligrafía japonesa. De esta manera, se está avanzando en el entendimiento mutuo entre los pueblos de Argentina y Japón (Embajada de Japón en la Argentina, 2018).

La colectividad de ese país en la Argentina (los nikeis) asciende a 65.000 personas.

En los dos últimos años, las actividades culturales de Japón en la Argentina se han visibilizado aún más, y enriquecido desde los más diversos ámbitos.

Con el convencimiento que la cultura es una de las tantas formas de entendimiento entre los pueblos, y observando el interés creciente por parte de diseñadores argentinos hacia el arte japonés, es que este ensayo pretende explorar la cultura japonesa con el fin de exponerla en estos escritos universitarios. Para ello, además de emplear bibliografía pertinente, se entrevistó a la filósofa nipona Mami Goda, quien reside en nuestro país desde hace poco más de ocho años.

Mami Goda nació en Japón, estudió Filosofía en la prestigiosa universidad francesa ParisSorbonne y en la École du Louvre, se especializó en reflexión sobre el arte. Trabajó en casas de subasta en las ciudades de París y en Tokio. En Buenos Aires es profesora, intérprete y traductora de japonés. Desde el año 2013 escribe para el periódico de la colectividad japonesa La Plata Hochi y ha colaborado en distintos medios gráficos y proyectos e intercambios de arte entre Argentina y Japón.

-MV: ¿Qué huellas del diseño japonés encuentras en la Argentina en la actualidad?

-MG: Hay mucho interés por lo que hay detrás del arte japonés: la filosofía, el pensamiento, la mirada hacia la naturaleza, la cultura. 
-MV: ¿Cómo interpretarías ese interés a partir de la filosofía? Por toda la crisis de occidente se puede pensar que estamos metidos en un consumismo desenfrenado... ¿Cómo lo expresarías desde la filosofía japonesa?

-MG: En Japón no tenemos esta mirada del dualismo entre el bien y el mal, lo correcto e incorrecto o lo bello y lo feo. En Japón hay una ambigüedad en la forma de ser, de ver las cosas, de hablar y de cómo es una belleza. Algo bello puede ser imperfecto.

-MV: Me interesa hablar de algunos conceptos que son clave en la cultura japonesa. Por ejemplo el de vacío ¿Los occidentales le tenemos horror al vacío, siempre hay algo que llenar... ¿cómo entiende este concepto un japonés?

-MG: Para nosotros el vacío es algo positivo. En el sentido que da todo un campo libre para la interpretación. El vacío es posibilidad, es algo que te tranquiliza, que da paz. Como algo meditativo, el vacío es silencio.

-MV: ¿Y cómo lo ves reflejado en la arquitectura o en el diseño de interiores?

-MG: Por ejemplo, si ves los objetos de la marca Muji no hay dibujos, motivos, es todo muy simple. Kenya Hara el Director artístico y diseñador japonés de la cadena de tiendas Muji, También es el Director artístico de la Japan House que se construyó en San Pablo. Él muestra dos fotos donde explica la diferencia entre dos cuchillos: uno es alemán, con un diseño muy funcional y otro un cuchillo japonés. El alemán tiene un diseño ergonómico, muestra cómo se encaja directo en tu mano. Es decir, tu mano no tiene que buscar cómo tiene que tomarlo, no tienes que pensar. El cuchillo japonés en cambio, es como muy finito, largo. Él lo toma como ejemplo para decir que el espacio del cuchillo alemán está lleno en cambio el japonés está vacío. Este último, dependiendo de cómo lo tomas, cambia la forma de cortar, qué cosa tienes que cortar. Si usas la punta para cortar es para hacer un fileteado del pescado crudo. Si lo utilizas más cerca de la mano, cortas más grueso. Kenya Hara afirma que es blanco porque es la función la que tienes que llenar al emplearlo.

-MV: ¿Cómo vinculas al kimono con el vacío?

-MG: Es justamente eso, tienes que decidir cómo te lo pones, cómo lo armas. Hay muchos diseñadores de la moda que construyen a partir de la forma de un maniquí, en cambio un kimono no. Es una forma única y un talle único que se adapta a cualquier cuerpo. Esto se está viendo acá también.

Es la técnica de furoshiki. Es una sola tela cuadrada, hay muchas maneras de envolver... es un poco como ésta: hay un modo para envolver una botella, dos botellas, una caja cuadrada, una caja redonda...en ese sentido la tela es vacía, es decir, tiene posibilidades. El kimono también...tiene un corte tan simple...en ese sentido entendemos el vacío.

En el diseño de Jardín también se aplica el vacío, lo mismo que en el Zen. Existe un vínculo muy fuerte de ese concepto dentro de toda la cultura.

-MV: El arte japonés fue fuente de inspiración para muchos artistas del siglo XIX y principios del XX, especialmente en aquellos rupturistas. La asimetría del arte japonés contrastaba con la usanza artística occidental y funcionaron como prácticas manumisoras de las convenciones de entonces... ¿Por qué crees que en la actualidad vuelve a impactar fuertemente la estética japonesa en el Diseño Occidental? ¿Esto lo ves también en el arte? 
-MG: Efectivamente, en la época del impresionismo fue muy fuerte la influencia del arte japonés.

-MV: ¿Por qué crees que esa influencia fue tan importante?

-MG: Porque el comercio de Japón estaba cerrado y se abrió en 1868. A partir de allí empezamos a trabajar con toda la traducción de los conceptos occidentales y a participar de la exhibición mundial. En ese momento, los occidentales comenzaron a conocer el arte japonés, especialmente los grabados ukiyo-e que influenciaron mucho al impresionismo, como así también la caligrafía. Lo vemos en las pinceladas, en los trazos. En la actualidad es muy fuerte esta influencia, ahora hay mucha gente que toma cursos de caligrafía, yo creo que el pintor argentino Eduardo Stupía trabaja mucho también con ese tipo de trazos.

-MV: ¿Crees que el sentido introspectivo y de interrelación entre el hombre y la naturaleza que se ve reflejada en la cultura japonesa es lo que impacta en la actualidad en occidente? Lo veo como una necesidad de búsqueda hacia un diseño más minimalista por parte de los occidentales. Algo que va más allá de lo puramente estético.

-MG: Sí, la imperfección o lo incompleto. Todo lo incompleto (que en occidente puede sonar como negativo) en Japón siempre existió. El arte del inacabamiento, que deja siempre un rincón vacío.

-MV: Y ese rincón vacío ¿que vendría a representar?

-MG: La posibilidad y un mundo infinito. Si llenas un espacio, se completa y se marca un límite. Si dejas un rincón vacío queda abierto.

Le pido que me de otro ejemplo del impacto del Zen en el diseño.

-MG: En la cerámica por ejemplo, se ve la influencia de lo japonés. Los alfareros occidentales contemporáneos trabajan de manera muy japonesa. Es decir, no ya con tazas blancas brillantes, sino con una estética más sobria.

-MV: Lo que dices me recuerda a lo que Tanizaki decía en El elogio de la sombra, ese maravilloso ensayo que explica la estética japonesa. Allí, el escritor expresa su manifiesta predilección por la vajilla de laca antes que la cerámica, si bien destaca que: "una vajilla de cerámica no es nada desdeñable, es cierto, pero a las cerámicas les faltan las cualidades de sombra y profundidad de las lacas" (Tanizaki, 2016, p. 35).

MG: Esto también se fue perdiendo por que nuestro país se occidentalizó. Hubo mucha modernización, hay una vuelta en la actualidad en Japón a volver lo clásico, a lo propio a lo rural.

-MV: Hoy se habla mucho de la identidad en América Latina y en Occidente en general. ¿Ustedes también sienten la necesidad de esa búsqueda de la propia identidad?

-MG: Sí, creo que sí. Fue muy fuerte el impacto del terremoto del norte en los japoneses. A partir de allí se vio una influencia en el arte de la cultura regional de cada uno. No sé si buscan la identidad japonesa, sino más bien la identidad del lugar de donde viene cada uno, más bien localista, de un pueblo o región. 
-MV: ¿Cómo lo ves desde lo filosófico?

-MG: Japón es un país insular, por su misma geografía, hay volcanes, siempre hubo terremotos, inmediatamente se producían incendios y siempre se perdía todo y se volvía a construir. Esto tiene que ver con el ciclo de las cuatro estaciones. La idea que una estación se va, pero después vuelve. Por esto del terremoto los japoneses, creo, volvieron a recordar esto de lo impermanentes que somos.

Seguimos hablamos del concepto de vacío. Pero luego le pregunto acerca de otros que están muy presentes en su cultura: el instante, la ambigüedad y la vulnerabilidad.

Le pido que haga referencia a esos conceptos vinculados al diseño, el arte la estética y a la filosofía.

La filósofa entonces vincula al concepto de instante directamente con la poesía Haiku. Para aclararlo recita una conocida poesía muy breve de (5, 7, 5 sílabas). Su voz resuena como un tintineo en las tazas de café del bar porteño donde se produjo el encuentro:

-MG: "El estanque antiguo

Salta una rana

El ruido del agua"

Tengo delante la copia de una vieja publicación que reproduce parte de una conferencia de Jorge Luis Borges llamada La Poesía del Japón que dictó en la Asociación Japonesa de la Argentina (imagino que sucedió a fines de los años setenta o principios de los ochenta), porque la misma carece de información precisa. Leo: (...) "parece que siempre los japoneses han sentido eso. Siempre han vivido en la idea de la brevedad" (s/f.) Luego, en otra página el mismo Borges continúa explicando el significado del concepto de instante:

Del estanque ha saltado una rana, sobre la gran campana se ha posado una mariposa. Hay ese instante. Ese instante vendría a ser como una revelación, un momento en el cual el sujeto, el yo, (el yo que según la filosofía budista es ilusorio), nuestra conciencia se identifica con la experiencia, y eso es ya poesía. Ahora sé que hay una relación, por lo menos siempre se afirma, entre el Budismo Zen y el Haiku... (Conferencia de Jorge L. Borges, La Poesía del Japón, s/f.).

Y luego, también refiriéndose al Haiku, Borges remata:

(...) esa poesía es del presente, de un presente que sí es precioso. Yo diría que esto es lo esencial del Haiku, por lo menos para este mero occidental que soy yo. El hecho de una poesía que sabe que el presente es precioso y que aprecia cada presente y que no piensa que eso se perderá y que eso formará parte del olvido. No, cada presente es precioso y es aceptado con gratitud. Y eso vendría a explicar el Haiku mejor que razones de orden fonético (...) es decir el goce del presente, el apreciar este presente. Ello vendría a ser una justificación. Y eso vendría a ser una justificación en el Budismo Zen y en el Haiku (Conferencia de Jorge L. Borges, La Poesía del Japón s/f.). 
-MG: Por ejemplo, la flor del cerezo que florece y enseguida se cae. Eso es efímero. Lo vemos como una forma de aceptación. No como algo negativo.

Respecto del concepto de ambigüedad, Goda explica que: "una taza rota no es fea sino que puede ser algo bello". Se rompe algo, la fisura se prepondera y hay una forma de resaltarlo que puede ser algo bueno, bello". Y aquí hace referencia al arte del kintsugi. Esta técnica implica reparar piezas rotas con materiales como el oro, platino o plata. La belleza reside en la originalidad de la misma y expresan su historia.

Durante una pausa de la entrevista y para ampliar el significado, leo al respecto en un artículo en la web que: “(...) entre sus principales influencias está el término estético wabi-sabi, que llama a ver la belleza en la imperfección, así como en los conceptos mottainai, que expresan lamentación cuando algo se desperdicia; y mushin, la aceptación del cambio" (Olguín, 2017).

-MV: Contrario a la mirada occidental, que busca la eterna juventud, la perfección.

En relación al concepto de vulnerabilidad, me advierte que ellos lo ven como una fortaleza, contrario a nuestra concepción occidental en la que la percibimos como debilidad.

Respecto de la ambigüedad, Goda explica que: "En la comunicación existe esta ambigüedad también. El japonés nunca dice No. Por eso a veces es difícil comunicarse con él” (Comunicación personal, 7 de diciembre de 2017).

-MV: ¿Por qué no dicen que no?

-MG: Para dejar pasar la situación. Para evitar el conflicto.

-MV: ¿Cómo sabe tu interlocutor que le están diciendo que no?

-MG: (risas) el otro tiene que adivinarlo.

-MV: Algo así como: L'ambasciator non porta pena

-MG: Sí, (ríe) eso exactamente.

-MV: ¿Cuál es la principal diferencia con el sentir estético occidental?

-MG: Si para la estética occidental -en el sentido de la regla de oro de los griegos- está dada por la simetría, la proporción y la perspectiva; para la japonesa es lo contrario: la asimetría, sin perspectiva, no se toma en cuenta la perspectiva.

-MV: ¿Cómo lo expresarías?

-MG: Creo que está vinculado con la naturaleza. Tiene que ver con la forma en que percibimos la naturaleza. Nosotros la vemos en capas [sic]: 70\% de la tierra en Japón es naturaleza: montañas y bosque (sin considerar las grandes ciudades). Antes si ibas a Japón a pasear era todo bosque. Insiste: "como en capas", no había una perspectiva, como ustedes que tienen la pampa, por ejemplo, eso te da una perspectiva. Esa apertura del paisaje que en Japón nunca hubo.

Hablamos de Tanizaki, de la arquitectura tradicional japonesa y de la búsqueda de la sombra “(...) por eso, cuando iniciamos la construcción de nuestras residencias, antes que 
nada desplegamos dicho tejado como un quitasol que determina en el suelo el perímetro protegido del sol, luego, en esa penumbra, disponemos la casa" (Tanizaki, 2016, p. 41).

Goda cuenta que actualmente en la arquitectura japonesa contemporánea hay mucha luz. Todavía en las zonas rurales se mantiene el estilo de la casa tradicional: con la puerta característica denominada shōji y sus marcos de madera y papel washi que funcionan como divisores. Lo vincula con la geografía y el clima más que con el pensamiento.

Mi mente vuelve al libro El elogio de la sombra:

Pero nosotros, no contentos con ello, proyectamos un amplio alero en el exterior de esas estancias donde los rayos el sol entran ya con mucha dificultad, construimos una galería cubierta para alejar aún más la luz solar. Y, por último, en el interior de la habitación, los shōji no dejan entrar más que un reflejo tamizado de la luz que proyecta el jardín. Ahora bien, precisamente esa luz indirecta y difusa es el elemento esencial de la belleza de nuestras residencias (Tanizaki, 2016, p. 43).

-MV: Los shōji, que dicho sea de paso, se pusieron de moda en la actualidad en el diseño de interiores minimalista en muchos países occidentales (por supuesto elaborados con otros materiales) ¿es algo que también se lo vincula al concepto de ambigüedad para ustedes? Por ejemplo, un pasillo puede ser un pasillo y luego transformarse en un cuarto.

-MG: Sí, exactamente. Este pasillo puede ser parte del jardín, pero también es como una terraza, o un balcón, como que pertenece afuera pero también es parte de un cuarto.

-MV: ¿En el diseño, es más bien funcional el concepto de ambigüedad?

-MG: Tiene dos sentidos. Esta parte es para protegerse de la lluvia y también funcional para que la gente pueda circular. La casa tradicional japonesa (como es muy húmedo Japón y hace mucho calor) está hecha pensando en los hombres dentro de un mundo rural.

-MV: ¿Qué sería un objeto bello para un japonés?

-MG: Un objeto que no es simplemente un objeto sino algo que vive, Por ejemplo, una taza, que la tocas para sentir el contenido.

Inevitablemente, para comprenderlo vuelvo a Tanizaki (2016):

Cuando sostengo en el hueco de mi mano un cuenco de sopa, nada me resulta más agradable que la sensación de pesadez líquida, de vívida tibieza que experimenta mi palma. Es una impresión análoga a la que produce al tacto la carne elástica de un recién nacido (p.35).

La conversación atraviesa naturalmente conceptos ligados a los sentidos y al diseño.

Para Goda, el diseño, no es solo visual, sino que lo vincula también con lo táctil. En relación al kimono sostiene:

Kimono es solo kimono [sic], sigue siendo algo tradicional. Hay jóvenes todavía en Japón que practican la ceremonia del té y disfrutan de ir a pasear por el 
jardín y se peinan al modo tradicional y lo siguen respetando como algo tradicional (Comunicación personal, 7 de diciembre de 2017).

-MV: ¿Hay algo que cuando ves una prenda de un diseñador japonés lo mires y digas “esto lo hizo un diseñador japonés"?

-MG: Sí, el pliegue, el corte, la forma que me hace pensar en el primer estado de la materia prima, papel o tela. Como muy simple, la bidimensión. Juega con el concepto de vacío entre el cuerpo y la tela.

En occidente la materia pierde su identidad para obedecer al cuerpo. En cambio en Japón tiene su propia forma la ropa. Por eso también es difícil llevarlo.

Reviso, busco y lo veo claramente en los diseños de Kunihiko Morinaga, de Anrealage; en Rei Kawakubo de Comme des Garçons; en Issey Miyake; sólo por mencionar algunos ejemplos.

-MV: ¿Cómo es ese vínculo entre la naturaleza y el diseño en oriente? ¿Cómo se manifiesta? -MG: La naturaleza está muy incorporada en el diseño. Por ejemplo en la comida, comes sushi y hay una decoración (por ejemplo hay una hoja de pino) que se hace de acuerdo a los elementos de la estación en que estás. Hay mucho vínculo entre los diseños y la naturaleza, en la moda quizás no tanto desde la forma pero sí desde los estampados. Hay muchos motivos decorativos que se inspiran en los elementos de la naturaleza. Lo ves también en los estampados del kimono.

-MV: ¿Cuánto es importante la materialidad de un objeto? ¿Es más importante que el diseño?

-MG: El diseño tiene la misma importancia. Pienso en los dulces japoneses que tienen formas de flores, elementos de la naturaleza. Creo que el diseño, la materia prima empleada tiene concordar con la naturaleza, no contraponerse. Se deben integrar.

(Esto concuerda con lo expresado por Tanizaki (2016) cuando hace alusión a que los colores del Yōkan, son de una complejidad difícil de encontrar en un dulce occidental)

-MV: Eso es muy de la cultura japonesa... pero, en la actualidad... ¿Sigue esa tradición? ¿Existe un vínculo con el Budismo?

-MG: Creo que hay, no digo en todo, pero hay gente que vive así. La filosofía está integrada a las costumbres, hay una mezcla. Es una forma de vida.

Los mismos jóvenes japoneses se sorprenden de la propia tradición y estudian el diseño clásico japonés, estudian mucho el diseño, la historia y la arquitectura japonesa.

-MV: Estamos en un mundo en transición (incertidumbre incesante por el futuro, tensiones, diásporas, migraciones masivas, agotamiento ambiental, etc.) ¿Cómo crees que están respondiendo ante esta situación artistas y diseñadores japoneses actualmente y cómo lo han hecho en el pasado?

¿Cómo crees que se podría ayudar en esta transición? ¿Crees que el diseño puede colaborar en un entendimiento entre los pueblos, mejorar la comunicación? 
-MG: Creo que hay una tendencia tanto en el arte y en el diseño como así también en la cultura en general de tomar conciencia de lo originario. Y de manifestarlo tanto en la forma como así también en la materia, con un ingenio en la adaptación pero sin perder las raíces. Pienso que ésta búsqueda es la única seguridad que tenemos en un mundo en transición: a partir de la idea de cómo es cada uno, cómo le gusta vivir: con cierta comodidad espiritual y corporal, es pensar en el clima y el ámbito que nos rodea. Es una forma de reconciliarse, o estar en armonía con su propia vida. Diseñar es un poco como crear el propio refugio que ofrece una sensación de seguridad.

\section{Comunicación, diseño, blanco y vacío}

El diseñador japonés Kenya Hara en el prólogo de su libro White (2017) explica el vínculo de varios de los conceptos analizados a lo largo de este escrito y aclara que si bien su trabajo es el de diseñar, su campo profesional es el de las comunicaciones. Explica que ilustra circunstancias o condiciones más que cosas. Para él, el vacío (utsu) y lo completamente hueco (karappo) son algunos de los términos que consideró al tratar de comprender la naturaleza misma de la comunicación.

Esto resulta esencial para comprender que el arte del lenguaje japonés, está indisolublemente vinculado al diseño y a su filosofía. Para un japonés hablar y escuchar es un verdadero arte. Hara (2017) ejemplifica ese instante blanco-vacío como el momento justo antes de que comience una actuación de música o danza. Al respecto, insiste en que se asemeja al de la pureza de la hoja en blanco, lo define como un estado de tabula rasa, tanto para la audiencia, como para el intérprete.

Para el japonés, el vacío es posibilidad. Por ello, tanto la comunicación como las ideas emergen del vacío.

Respecto del vínculo de la cultura japonesa con la naturaleza, la creación y la filosofía Zen, algunos autores como Hara (2017) consideran que la sensibilidad japonesa probablemente esté más en sintonía con la conservación que con la novedad. En sus mismas palabras: "No me refiero a que los japoneses no sean creativos, sino creo que la innovación proviene de esa conciencia que trata de mantener las cosas tal como son. El poder de la transformación no es algo nuevo" (p. 70). Es decir, así como la naturaleza se modifica constantemente, se requiere mucha energía y armonía para preservar las cosas bellas, de modo que es fundamental observar esa transformación muy de cerca, para capturar su quietud mientras se defienden sus características inmutables y universales.

Como se ha podido observar, la filosofía impregna el diseño japonés y vincula cada uno de los conceptos analizados. El diseño japonés es vida, filosofía, arte, estética e innovación en estado puro. Puro como el blanco. En palabras de Hara (2017) "la simplicidad lleva al vacío, el espacio donde reside la mente de las personas, como hemos visto, es el origen de la comunicación y se refleja en cosas como el mito japonés" (p. 55).

\section{Bibliografía}

Borges, J. L. (1993). Siete Noches. Madrid: Fondo de Cultura Económica 
La Poesía del Japón Asociación Japonesa de la Argentina (s/f ).

Embajada de Japón en la Argentina, 2018. Recuperado de: http://www.ar.emb-japan.go.jp/ itpr_es/RelacionesBilaterales.html

Hara, J. (2017). White. Zürich: Lars Müller Publishers.

JVT 2006.7 -- Furoshiki the Flexible Wrapping Cloth. Recuperado de: https://www.youtube. com/watch?v=ERT_WSr0o3M

Olguin, B. "Farenheit Magazine", El arte japonés del 'Kintsugi' o la belleza de lo imperfecto 2 de agosto de 2017. Recuperado de: http://fahrenheitmagazine.com/cultura/centenarioarte-japones-kintsugi-belleza-imperfecto/

Shimizu, C. (1984). El arte Japonés. Buenos Aires: Paidós.

Tanizaki, J. (2016). El elogio de la sombra. Madrid: Siruela.

\begin{abstract}
Given the growing interest in Japanese culture in Argentina, this essay attempts to analyze the link between the concepts of moment, emptiness, ambiguity and vulnerability, to relate them to design, art, aesthetics, Zen philosophy and nature. The kimono, the characteristic door called shōji, its wooden frames and washi paper, Haiku poetry, white and shadows, are some of the examples that are addressed to explain it. It is also essential to understand that Japanese language is also inextricably linked to design and its philosophy. In Japanese culture, speaking and listening is a true art and the reason why this paper addresses the issue from not only the texts of Kenya Hara, Junichirō Tanizaki and Jorge Luis Borges, but also includes -necessarily- a dialogue between the author of this article and the Japanese philosopher Mami Goda, resident in Argentina for almost a decade.
\end{abstract}

Keywords: Japanese culture - emptiness - moment - ambiguity - vulnerability - design art - Zen philosophy - Haiku- kimono.

Resumo: Dado o crescente interesse pela cultura japonesa na Argentina, no presente ensaio tenta analisar a ligação entre os conceitos de instante, vazio, ambiguidade e vulnerabilidade, relacioná-los com design, arte, estética, filosofia zen e natureza. O quimono, a porta característica chamada shōji, seus quadros de madeira e papel washi, a poesia Haiku, branco e sombras, são alguns dos exemplos que são abordam-se para explicá-lo. Também é essencial entender que a linguagem japonês está intrinsecamente ligado ao design e à sua filosofia. Para um japonês falar e Ouvir é uma verdadeira arte. É por isso que o resumo aborda a questão não apenas dos textos do Quênia Hara, Junichirō Tanizaki e Jorge Luis Borges, mas também inclui-necessariamente- um diálogo entre o autor deste artigo e o filósofo nipona Mami Goda, residente na Argentina há quase uma década.

Palavras chave: Cultura japonesa - vazio - instantâneo - ambigüidade - vulnerabilidade design- arte - filosofia Zen-Haiku-kimono.

[Las traducciones de los abstracts fueron supervisadas por el autor de cada artículo] 